\title{
Implicit Constraint Enforcement for Rigid Body Dynamic Simulation
}

\author{
Min Hong ${ }^{1}$, Samuel Welch ${ }^{2}$, John Trapp ${ }^{2}$, and Min-Hyung Choi ${ }^{3}$ \\ ${ }^{1}$ Division of Computer Science and Engineering, Soonchunhyang University, \\ 646 Eupnae-ri Shinchang-myeon Asan-si, Chungcheongnam-do, 336-745, Korea \\ Min. Hong@UCHSC . edu \\ ${ }^{2}$ Department of Mechanical Engineering, University of Colorado at Denver and Health \\ Sciences Center, Campus Box 109, PO Box 173364, Denver, CO 80217, USA \\ \{Sam, jtrapp\} @ carbon. cudenver. edu \\ ${ }^{3}$ Department of Computer Science and Engineering, University of Colorado at Denver and \\ Health Sciences Center, Campus Box 109, PO Box 173364, Denver, CO 80217, USA \\ Min-Hyung. Choi@cudenver. edu
}

\begin{abstract}
The paper presents a simple, robust, and effective constraint enforcement scheme for rigid body dynamic simulation. The constraint enforcement scheme treats the constraint equations implicitly providing stability as well as accuracy in constrained dynamic problems. The method does not require ad-hoc problem dependent parameters. We describe the formulation of implicit constraint enforcement for both holonomic and non-holonomic cases in rigid body simulation. A first order version of the method is compared to a first order version of the well-known Baumgarte stabilization.
\end{abstract}

\section{Introduction}

Rigid body simulation is highly important in the modeling of various physical systems and has been comprehensively studied in electrical and mechanical engineering. Rigid body dynamics involves a variety of holonomic constraints such as ball-andsocket, hinges, sliders, universal joints, and contact constraints. In addition, nonholonomic constraints have been used in simulations of robots and cars. In rigid body dynamics, constraint enforcement is critical to guarantee successful simulations since small but accumulating constraint drift could cause instabilities. Constraint satisfaction through the use of Lagrange multipliers represents a challenging problem numerically as the resulting system of equations is a mixed ODE algebraic system. Early workers solved this problem by differenting the constraint equations replacing the algebraic equation with an ODE but the solutions exhibited numerical drift in the constraint error. Baumgarte's stabilization method [1] was introduced to reduce this numerical drift using parameter dependent stabilization terms and is still widely used today due to it's simplicity.

In this paper, we propose an implicit holonomic and non-holonomic constraint enforcement method for rigid body dynamics that provides numerical stability and accuracy without requiring ad-hoc stabilization parameter terms while providing the same asymptotic computational cost as the Baumgarte method. The approach is to implicitly expand the algebraic constraint in a Taylor series to the same order as the order of 
the numerical method used to integrate the ODE system. The Taylor expansion is implicit in the state variables thus the method has desirable stability characteristics.

\section{Related Work}

The seminal work of Baumgarte [1] included second order feedback terms to stabilize constraints and has been used successfully for many applications $[2,3,4,6]$. Numerous improved methods have appeared over the decades since Baumgarte presented his method but Baumgarte's stabilization is widely used due to its simplicity and the ease with which it is understood. The main drawback to Baumgarte stabilization is that the required parameters must be chosen in an ad-hoc manner. Another approach is poststabilization in which a correction is made to the state variables in order to minimize the constraint error. An example of this is Cline and Pai [5] who proposed a poststabilization approach for rigid body simulation. Post-stabilization requires additional computational load to reinstate the accuracy in the constraint and it can cause error in the motion of objects under complicated situations since the constraint drift reduction is performed independently from the conforming dynamic motions [7].

\section{Implicit Holonomic Constraint Enforcement}

In this section a simple implicit constraint enforcement method is presented and compared to Baumgarte's stabilization. It will be shown that a certain selection of Baumgarte parameters will result in the two methods being nearly identical for the holonomic case. A stability analysis will be presented and used as a guide to select parameters for Baumgarte's stabilization technique. Let $\Phi$ be the vector of holonomic constraints. The equations of motion for the holonomically constrained system are written as

$$
\begin{aligned}
& M \ddot{r}+\Phi_{r}^{T} \lambda=F \\
& J \dot{\omega}+\Phi_{\pi}^{T} \lambda=T-\tilde{\omega} J \omega \\
& \Phi(r, e)=0 \\
& \dot{e}=\frac{1}{2} G(e) \omega
\end{aligned}
$$

Where $r$ are the center of mass coordinates, e are the Euler parameters associated with the orientation of the rigid bodies, $\Phi_{r}$ and $\Phi_{\pi}$ are the constraint jacobians associated with $\mathrm{r}$ and the orientation, respectively and $\lambda$ is a vector containing the Lagrange multipliers. $\mathrm{M}$ and $\mathrm{J}$ are inertia matrices and $\tilde{\omega}$ is the skew-symmetric matrix associated with the angular velocity $\omega$. The matrix defining the time derivative of the Euler parameters and the angular velocity is given by:

$$
G(e)=\left\{\begin{array}{cccc}
-e 1 & e 0 & e 3 & -e 2 \\
-e 2 & -e 3 & e 0 & e 1 \\
-e 3 & e 2 & -e 1 & e 0
\end{array}\right\}
$$


Equations (1), (2) and (4) are discretized to first order as:

$$
\begin{gathered}
\dot{r}(t+\Delta t)=\dot{r}(t)+\Delta t M^{-1} F-\Delta t M^{-1} \Phi_{r}^{T} \lambda \\
r(t+\Delta t)=r(t)+\Delta t \dot{r}(t+\Delta t) \\
\omega(t+\Delta t)=\omega(t)+\Delta t J^{-1} T-\Delta t J^{-1} \tilde{\omega}(t) J \omega(t)-\Delta t J^{-1} \Phi_{\pi}^{T} \lambda \\
e(t+\Delta t)=e(t)+\frac{\Delta t}{2} G(e) \omega(t+\Delta t)
\end{gathered}
$$

The basic philosophy of the implicit constraint enforcement technique we use is to expand the algebraic constraint function in a Taylor series to the same order as the discrete equations. The resulting expansion is:

$$
\Phi(t+\Delta t)=\Phi(t)+\left\{\Phi_{r} \dot{\vec{r}}(t+\Delta t)+\Phi_{\pi} \omega(t+\Delta t)\right\} \Delta t=0
$$

Equations (6) through (8) may be used to eliminate the new time velocity terms to obtain the linear system that must be solved for the Lagrange multiplier:

$$
\left\{\Phi_{r} M^{-1} \Phi_{r}^{T}+\Phi_{\pi} J^{-1} \Phi_{\pi}^{T}\right\} \lambda=\frac{\Phi}{\Delta t^{2}}+\frac{1}{\Delta t}\left(\Phi_{r} \dot{\vec{r}}+\Phi_{\pi} \vec{\omega}\right)+\Phi_{r} M^{-1} F+\Phi_{\pi} J^{-1}(T-\tilde{\omega} J \vec{\omega})
$$

In this equation and in all equations that follow terms without explicit time dependence indicated are evaluated at old time. Once this system is solved for the Lagrange multipliers equations (6) through (9) are used to update the other variables. Note that this linear system is symmetric positive definite. Baumgarte's stabilization technique may be used for this system in the following way. First the constraint is stabilized by adding two terms to the second derivative with parameters $\alpha$ and $\beta$ as follows:

$$
\ddot{\Phi}+2 \alpha \dot{\Phi}+\beta^{2} \Phi=0
$$

A similar procedure is used to obtain the linear system to be solved for the Lagrange multipliers:

$$
\begin{aligned}
\left\{\Phi_{r} M^{-1} \Phi_{r}^{T}+\Phi_{\pi} J^{-1} \Phi_{\pi}^{T}\right\} \lambda & =\beta^{2} \Phi+2 \alpha\left(\Phi_{r} \dot{\vec{r}}+\Phi_{\pi} \vec{\omega}\right)+\Phi_{r} M^{-1} F \\
& +\Phi_{\pi} J^{-1}(T-\tilde{\omega} J \vec{\omega})+\left(\Phi_{r} \dot{\vec{r}}\right)_{r} \dot{\vec{r}}+\left(\Phi_{\pi} \vec{\omega}\right)_{\pi} \vec{\omega}
\end{aligned}
$$

As with the simple implicit method, once this system is solved for the Lagrange multipliers equations (6) through (9) are used to update the other variables. Baumgarte stabilization is often criticized because selection of the parameters $\alpha$ and $\beta$ is ad-hoc and problem dependent. Greenwood [9] suggests that both parameters should be proportional to the time step and a cursory examination of equations (12) and (10) indicates that the choices $\alpha=0.5 \Delta t^{-1}$ and $\beta=\Delta t^{-1}$ would result in our method and Baumgarte's stabilization differing by only the last two terms in equation (12). In what follows we analyze the stability of these schemes and use the result to guide us in a selection for the parameters $\alpha$ and $\beta$. In order to analyze the stability the schemes described above we lump the center of gravity and orientation variables into a single vector $\mathrm{q}$ and we consider a linearized system with linearized constraints with constraint gradient given by 


$$
\Phi_{q}(q)=\mathrm{B}
$$

The dynamic system will have only constraint forces as we wish to isolate the effect of constraint enforcement on stability. The discretized homogeneous equations of motion may be written as:

$$
\begin{aligned}
& \dot{q}(t+\Delta t)=\dot{q}(t)-\Delta t M^{-1} \mathrm{~B}^{T} \lambda \\
& q(t+\Delta t)=q(t)+\Delta t \dot{q}(t+\Delta t)
\end{aligned}
$$

Note that the mass matrix and inertia matrix are now lumped together. The linear system that must be solved for the Lagrange multiplier becomes.

$$
\mathrm{B} M^{-1} \mathrm{~B}^{T} \lambda=\frac{1}{\Delta t^{2}} \mathrm{~B} q(t)+\frac{1}{\Delta t} \mathrm{~B} \dot{q}(t)
$$

The Lagrange multipliers may now be eliminated and the resulting linear state-space form of the equations of motion is

$$
\left\{\begin{array}{c}
q(t+\Delta t) \\
\dot{q}(t+\Delta t)
\end{array}\right\}=X\left\{\begin{array}{c}
q(t) \\
\dot{q}(t)
\end{array}\right\}
$$

where

$$
\mathrm{X}=\left[\begin{array}{lll}
\mathrm{I}-M^{-1} \mathrm{~B}^{T} & \mathrm{~A}^{-1} \mathrm{~B} & \Delta t\left(\mathrm{I}-M^{-1} \mathrm{~B}^{T} \mathrm{~A}^{-1} \mathrm{~B}\right) \\
\frac{-1}{\Delta t} M^{-1} \mathrm{~B}^{T} & \mathrm{~A}^{-1} \mathrm{~B} & \mathrm{I}-M^{-1} \mathrm{~B}^{T} \mathrm{~A}^{-1} \mathrm{~B}
\end{array}\right]
$$

where $\mathrm{A}=\mathrm{B} M^{-1} \mathrm{~B}^{T}$. The condition for stability of this system of equations is

$$
\rho(\mathrm{X}) \leq 1
$$

where $\rho(X)$ is the spectral radius of the matrix $X$. A similar result may be derived for the Baumgarte method with the result

$$
\left\{\begin{array}{c}
q(t+\Delta t) \\
\dot{q}(t+\Delta t)
\end{array}\right\}=\Lambda\left\{\begin{array}{c}
q(t) \\
\dot{q}(t)
\end{array}\right\}
$$

Where

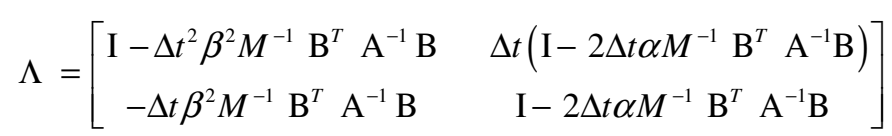

The condition for stability for the Baumgarte method is

$$
\rho(\Lambda) \leq 1
$$

Note that the choices $\alpha=0.5 \Delta t^{-1}$ and $\beta=\Delta t^{-1}$ would result in the Baumgarte stabilization method having the same spectral radius as our implicit method as the differing 
terms were discarded in the linearization. In order to investigate the stability of the methods further we consider the simple problem of a planar double pendulum with links modeled by point masses. The equations of motion are written using Cartesian coordinates thus we have two constraints that force the link lengths to be constant.

The link lengths considered are unity as are the point masses. The constraints are linearized about a co-linear position and the resulting constraint jacobian for this linearized system is

$$
B=\left[\begin{array}{cccc}
2 & 0 & 0 & 0 \\
-2 & 0 & 2 & 0
\end{array}\right]
$$

For this case, $\rho(X) \leq 1$ for all $\Delta t$ thus the implicit constraint enforcement method is unconditionally stable. The stability behavior of the Baumgarte's stabilization depends on the selection of the parameters $\alpha$ and $\beta$. For example, if we select $\alpha, \beta=10$ the resulting stability limit is $\Delta t \leq 0.83$. If we select parameters $\alpha=\Delta t^{-1}$ and $\beta=\Delta t^{-1}$ which give us a critically damped response with time constant proportional to $\Delta t$ we will find that the system is unstable. Therein lies the difficulty in parameter selection for Baumgarte's stabilization technique. Improper choices for the parameters $\alpha$ and $\beta$ often results in time step limitations due to stability or in the time constants associated with constraint satisfaction being too slow. One of the advantages of the simple implicit constraint enforcement scheme described in this paper is that the search for a good choice of parameters need not be undertaken and, as we will show, the method produces accurate results. For the comparisons in the rest of this paper we will use the parameter selections $\alpha=0.5 \Delta t^{-1}$ and $\beta=\Delta t^{-1}$ for the reasons cited above. We note that these parameter selections specify a damping coefficient of 0.5 and a time constant on the order of the time step for constraint satisfaction.

\section{Implicit Non-holonomic Constraints Enforcement}

Non-holonomic constraints typically restrict velocities for points on objects. In the development that follows the implicit constraint enforcement technique is developed using functional dependences on the center of gravity coordinates only. Extension to dependence on orientation coordinates follows identical development. The equations of motion for the non-holonomic constraint can be written as

$$
{ }^{N} \Phi_{r}(r) \dot{r}=v_{o}
$$

Following our earlier philosophy the constraint written at new time is

$$
{ }^{N} \Phi_{r}(r(t+\Delta t)) \dot{r}(t+\Delta t)=v_{o}(t+\Delta t)
$$

Next a Taylor series expansion is written to the same order as the discrete equations:

$$
\begin{aligned}
{ }^{N} \Phi_{r}(r(t+\Delta t)) \dot{r}(t+\Delta t) & ={ }^{N} \Phi_{r}(r(t)) \dot{r}(t)+\left({ }^{N} \Phi_{r}(r(t)) \dot{r}(t)\right)_{r}(r(t+\Delta t)-r(t)) \\
& +\left({ }^{N} \Phi_{r}(r(t)) \dot{r}(t)\right)_{\dot{r}}(\dot{r}(t+\Delta t)-\dot{r}(t))
\end{aligned}
$$


where the subscript $r$ indicates partial differentiation with respect to $r$. After simplification we have:

$$
{ }^{N} \Phi_{r}(r(t+\Delta t)) \dot{r}(t+\Delta t)=\left({ }^{N} \Phi_{r}(r(t)) \dot{r}(t)\right)_{r}(r(t+\Delta t)-r(t))+{ }^{N} \Phi_{r}(r(t)) \dot{r}(t+\Delta t)
$$

Equation (7) may be used to eliminate $r(t+\Delta t)$ to obtain

$$
{ }^{N} \Phi_{r}(r(t+\Delta t)) \dot{r}(t+\Delta t)=\Delta t\left({ }^{N} \Phi_{r}(r(t)) \dot{r}(t)\right)_{r} \dot{r}(t+\Delta t)+{ }^{N} \Phi_{r}(r(t)) \dot{r}(t+\Delta t)
$$

The first term may be written to second order replacing $\dot{r}(t+\Delta t)$ with $\dot{r}(t)$ resulting in

$$
{ }^{N} \Phi_{r}(r(t+\Delta t)) \dot{r}(t+\Delta t)=\Delta t\left({ }^{N} \Phi_{r}(r(t)) \dot{r}(t)\right)_{r} \dot{r}(t)+{ }^{N} \Phi_{r}(r(t)) \dot{r}(t+\Delta t)
$$

Equation (6) may be used to eliminate $\dot{r}(t+\Delta t)$ and the result is substituted into Equation (32) to obtain the linear system that must be solved for the Lagrange multiplier:

$$
{ }^{N} \Phi_{r} M^{-1 N} \Phi_{r}^{T} \lambda=\frac{1}{\Delta t}{ }^{N} \Phi_{r} \dot{r}+{ }^{N} \Phi_{r} M^{-1} F+\left({ }^{N} \Phi_{r} \dot{r}\right)_{r} \dot{r}-\frac{v_{o}(t+\Delta t)}{\Delta t}
$$

Note that we obtain the desirable symmetric positive definite linear system as we did with the holonomic case. The development for the case with orientation variable dependence of the constraints is identical. Once this system is solved for the Lagrange multipliers equations (6) through (9) are used to update the other variables. Baumgarte's stabilization for the non-holonomic case begins with the constraint function written as $g(t)=0$. Baumgarte's stabilization may be expressed as (Greenwood [8]).

$$
\dot{g}+2 \alpha g+\beta^{2} \int_{o}^{t} g d t=0
$$

For our case we write

$$
g(t)={ }^{N} \Phi_{r}(r) \dot{r}-v_{o}=0
$$

and Baumgarte's stabilization becomes

$$
{ }^{N} \Phi_{r} M^{-1}{ }^{N} \Phi_{r}^{T} \lambda=-\dot{v}_{o}+\left({ }^{N} \Phi_{r} \dot{r}\right)_{r} \dot{r}+{ }^{N} \Phi_{r} M^{-1} F+2 \alpha\left({ }^{N} \Phi_{r} \dot{r}-v_{o}\right)+\beta^{2} \int_{0}^{t}\left({ }^{N} \Phi_{r} \dot{r}-v_{o}\right) d t
$$

Note again the appearance of extra terms in when using Baumgarte's stabilization and in particular the appearance of the integral that must be calculated. In the results that follow we evaluate the integral with a first-order method consistent with the method used to integrate the ODE's.

\section{Experiments}

To verify the accuracy of the proposed holonomic and non-holonomic implicit constraint enforcement, we performed a three-link rigid bar simulation in Fig. 1. Three separate rigid bars are connected by three spherical joints. The non-holonomic 


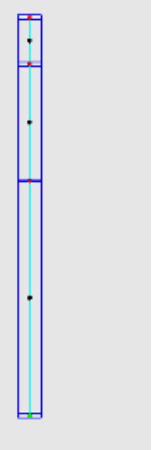

(a) Initial state of rigid bar

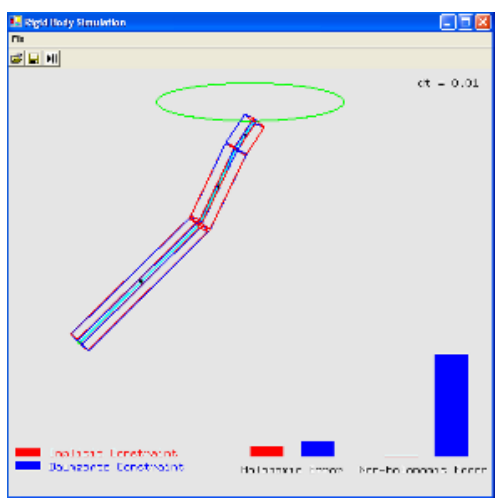

(b) The motion of rigid-bar during simulation

Fig. 1. The three-link rigid bar simulation with holonomic and non-holonomic constraints. The green circle indicates the trajectory of the point where the non-holonomic constraint is applied.
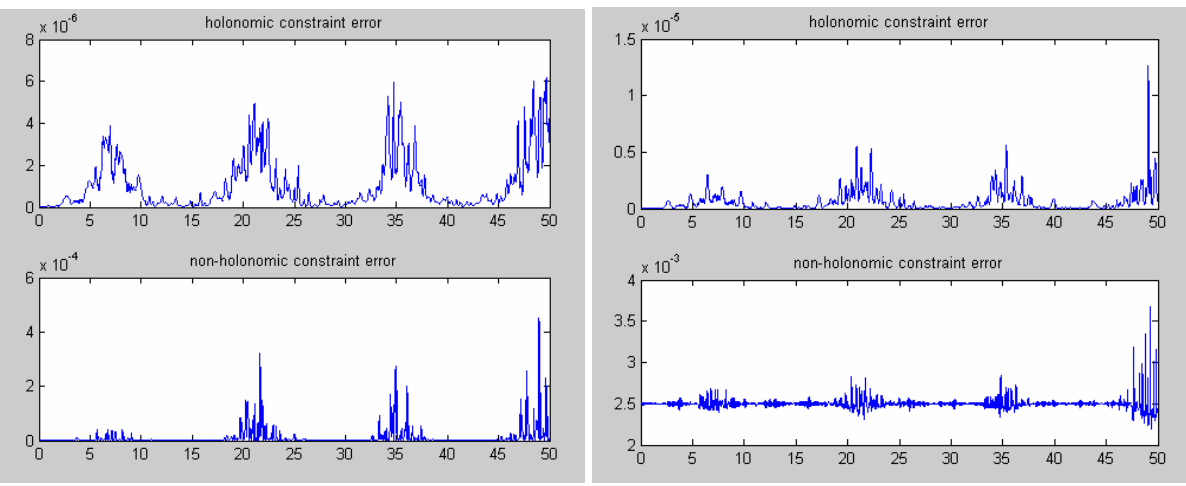

(a) Implicit constraint error using $\Delta t=0.01$

(b) Baumgarte constraint error using $\Delta t=0.01$
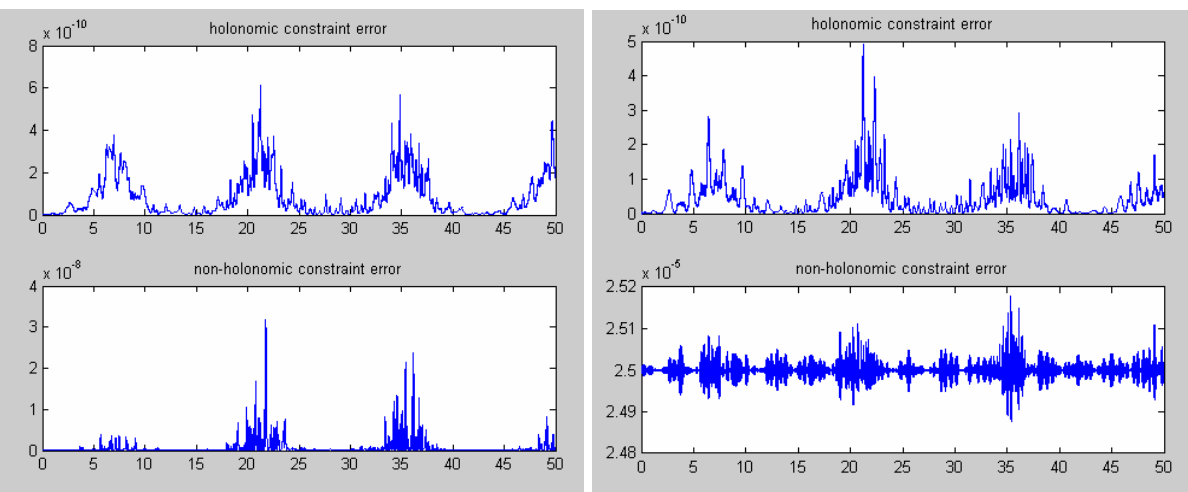

(c) Implicit constraint error using $\Delta t=0.001$ (d) Baumgarte constraint error using $\Delta t=0.001$

Fig. 2. Comparison of holonomic and non-holonomic constraint errors for three-link rigid bar simulation 
constraint is applied on the top end of the first link. The point in the end of this link has a circular velocity prescribed. The constraint is integrable but we treat it as a nonholonomic constraint for illustration purposes. The prescribed velocity results in the circular movement of the end point of the link in the x-y plane.

We recorded the holonomic and non-holonomic constraint errors over a time duration of 50 seconds and the results are shown in Fig 2. The Baumgarte stabilization parameters used are $\alpha=0.5 \Delta t^{-1}$ and $\beta=\Delta t^{-1}$. The results shown indicate somewhat better results for the non-holonomic constraint errors with our method and similar results for the holonomic constraint errors. These results are typical of what we have observed and we note that if we do not include the integral in the Baumgarte method the results are less accurate.

\section{Conclusion}

This paper describes an implicit constraint enforcement method for rigid body simulations with both holonomic and non-holonomic constraints. Our implicit constraint enforcement method has several advantages. This method is implicit thus it has desirable stability characteristics. The method does not require problem dependent parameters and does not require an integral computation for the non-holonomic case. The method is simple to implement, requiring fewer terms than Baumgarte stabilization. The method results in a symmetric positive definite linear system identical to that in Baumgarte stabilization. The method is easily extended to second order and the second order method will appear in future work.

\section{References}

1. J. Baumgarte, Stabilization of constraints and integrals of motion in dynamical systems, Computer Methods in Applied Mechanics, pp. 1-16, 1972.

2. D. Baraff, Linear-time dynamics using Lagrange multipliers, In proceedings of SIGGRAPH 1996, ACM Press / ACM SIGGRAPH, Computer Graphics Proceedings, pp. 137-146, 1996.

3. R. Barzel, and A. Barr, A modeling system based on dynamic constraints, Computer Graphics, 22(4), pp. 179-188, 1988.

4. J. C. Platt, and A. Barr, Constraint methods for flexible models, Computer Graphics, 22(4), pp. 279-288, 1988.

5. M. B. Cline, and D. K. Pai, Post-stabilization for rigid body simulation with contact and constraints, In proceedings of the IEEE International Conference on Robotics and Automation, pp. 3744-3751, 2003.

6. H. Jeon, M. Choi, and M. Hong, Numerical stability and convergence analysis of geometric constraint enforcement in dynamic simulation systems, In proceedings of the International Conference on Modeling, Simulation and Visualization Methods, pp. 207-213, 2004.

7. M. Hong, M. Choi, S. Jung, S. Welch, and J. Trapp, "Effective constrained dynamic simulation using implicit constraint enforcement," in Proceedings of the 2005 IEEE International Conference on Robotics and Automation, pp. 4531-4536, 2005.

8. D. Greenwood, Advanced Dynamics, Cambridge, 2003 